\title{
Nanometric $z$-Profiling of Bio-Chromophore Layers by DRLS
}

\author{
S.A. Popescu And A. Peled* \\ Photonics Laboratory, Holon Institute of Technology, Holon 5810201, Israel \\ (Received January 10, 2020; in final form April 6, 2020)
}

\begin{abstract}
The differential reflected light scattering technique provides a nondestructive, far field optical back-scattering measurement method with $z$-thickness profiling capabilities over large areas. The phenomenological theory and experimental examples of thickness profiling and structural evaluation of photodeposited organic materials layers assisted by bio-chromophores are described in this work.
\end{abstract}

DOI: 10.12693/APhysPolA.137.1163

PACS/topics: differential reflected light, nanolayers, light scattering, optical nanoscopy, chromophores, photodeposition

\section{Introduction}

Surprisingly, only a few papers were published until now in the literature on optical diagnostic techniques using methods of optimized high contrast photon backscattering processes from thin nanolayer materials deposited on transparent waveguides serving as substrates [1-4] for thickness profiles evaluation. This is probably due to the reason that most film thickness measurements are made traditionally by simple perpendicular optical transmission, which is a satisfactory sensitive technique for micrometric layers but not always good enough for nanometric layers. In contrast, the DELI technique based on the evanescence phenomenon developed by us formerly $[5-8]$ and extended here particularly to an analogous technique called differential reflected light scattering (DRLS) method proves suitable to analyze the thickness profiles of extremely thin nanolayers and nanoparticles as obtained for instance by photodeposition in our laboratory [7].

In the DRLS technique the nanometric thickness sample is optically illuminated from above by a visible spectra diffused light illuminator at an optimal oblique angle $\theta_{C}$ to obtain best contrast scattered evanescent light images features. The backscattered light intensity images are captured by a wide field optical microscope equipped with a CMOS camera connected to a computer for analyzing the required $1 \mathrm{D}, 2 \mathrm{D}$, and $3 \mathrm{D}$ thickness profiles of the deposited patterns and even their internal structures.

\section{Optical measuring procedure}

In this investigation, images of photodeposited biochromophores nanolayers on polymethyl-methacrylate (PMMA) were captured by an optical microscopy system schematically shown in Fig. 1. To evaluate the $z$-thickness profiles of the deposited nanometric layers

*corresponding author; e-mail: peleddd@gmail.com

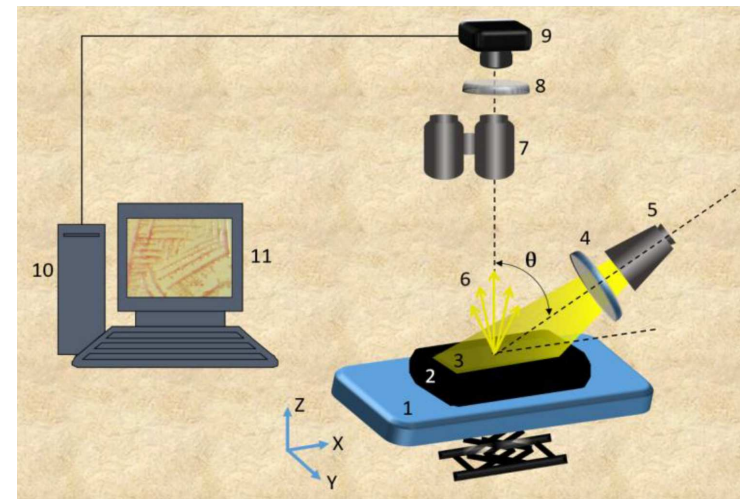

Fig. 1. Schematic illustration of the microscope setup: $1-z$-stage, $2-x-y$ shifting holder, $3-$ substrate with deposited material, 4 - $p$-polarizer, 5 - LED lamp, 6 - scattered light distribution, 7 - zooming microscope, 8 - optical density filter, 9 - CMOS camera, $10-\mathrm{PC}, 11-\mathrm{PC}$ display, $\theta-$ angle of beam incidence on the sample surface.

by DRLS, the sample illumination was supplied by a diffuse white LED lamp. The thickness profiles evaluation by the DRLS method is presented in the following sections.

\section{DRLS technique}

\subsection{The layers illumination schematics}

Figure 2 depicts a thin layer cross-section, where a light beam hits the sample on the upper air/surface interface at an angle $\theta$ and is back reflected and also penetrates by refraction into the nanolayer. Inside the layer, the beam can be scattered, reflected and refracted many times by the nanoparticles before it arrives to the transparent underlying substrate/waveguide where it is finally absorbed in the black slab interface positioned below the substrate. The $z$-backscattered intensity $I_{z}$ is proportionally weaker from thinner layer zones towards the microscope since they contain less scattering atoms. The optical images recorded by the microscope from the light scattered 


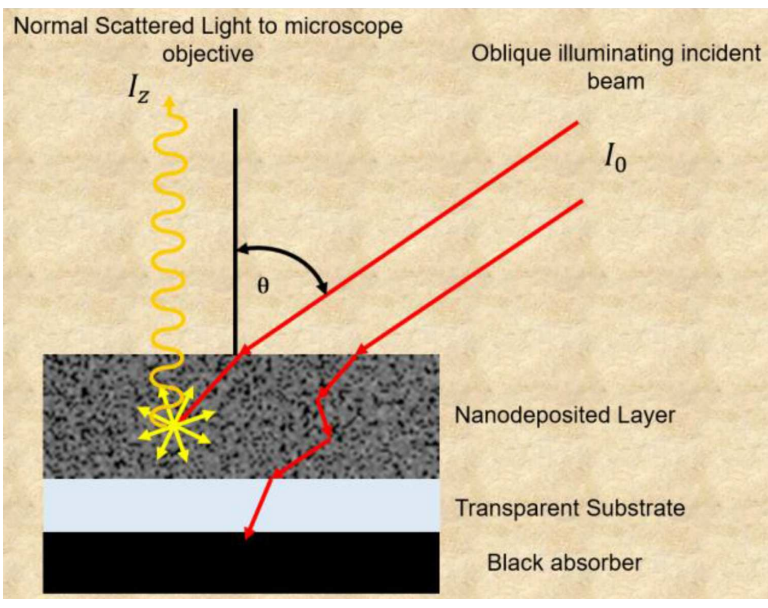

Fig. 2. Cross-section illustration of the light rays propagating in a thin layer composed of random packed nanoparticles.

from the layer depends on the deposited material reflection, scattering and absorbing properties and the degree of polarization of the illuminating beam such as $s$ - or $p$-polarization or mixed polarized light.

Experimentally, we found that under optimal skew observation angles the optical contrast of the optical microscope images is good enough to observe the nanolayers particles thickness profiles for various nanometric depths. The ideal Fresnel equations indicate how much light is reflected and transmitted at a surface as a function of the angle of incidence $\theta_{i}$ and the refractive indices of the incidence and transmissivity of the media [9]. The various optical parameters such as refractive indices of air, deposited layers surface roughness, the interfaces between the particulate grains, transparent substrate and backing black slab optical absorption and scattering properties pose a very complex situation for analytical or numerical solution for the general case [2, 3]. Moreover, light polarization makes the calculations more complicated for oblique light incidence onto the various surfaces [4]. The classical equations of Fresnel for instance predict a nil $p$-polarized specular reflection at about $\approx 56^{\circ}$ incidence angle for air/glass or air/polymer interfaces, corresponding to the Brewster angle. This angle is thus preferred for obtaining microscope images with higher contrast of the backscattered photons from the deposited layer internal particles/grains vs. the upper and lower interfaces of the specular reflection from the nanolayer and substrate. At such an optimal incidence angle $\theta_{C}$, a maximum fractional power mode of scattered light $I_{z} / I_{0}$ towards the microscope objective is usually attained from the illuminating beam impinging on the sample surface providing best height contrast images. This kind of differential scattering contrast phenomenon is encountered well by any observer who checks the cleanliness of eyeglasses optical surfaces for any greasy or nanoparticulate deposition by checking them at an appropriate grazing illumination angle with respect to the glass surface.
In the DRLS model it is assumed phenomenologically that the scattered power from the material deposited on the transparent substrate/waveguide depends in a first approximation to the number of deposited nanoparticles on the substrate surface, similar to the assumption in the DELI model $[5-8,10]$. In the bare, non-deposited areas of the flat substrate surface, an almost complete transmission occurs by down refraction of the illuminating beam and very little light emerges up due to the black absorbing slab placed underneath the transparent substrate.

The perpendicular optical image of the emanating scattered light $I_{z}$ from the deposited layer on top of the transparent substrate/waveguide through its upper face is captured by a zooming microscope, type Leica Wild M3Z equipped with a CMOS camera, with spectral peak response at $555 \mathrm{~nm}$ wavelength. By the DRLS method, from the 2D nanolayers images captured by the CMOS camera, the normalized integrated optical density (NIOD) is obtained by integration over an appropriate image area and normalized into an effective gray level, i.e.

$$
\mathrm{NIOD}=\frac{\iint_{S} D(x, y) \mathrm{d} s}{D_{\max } S} .
$$

$D(x, y)$ is the gray level value of each pixel (0-255), $S$ is the area of the sampled $2 \mathrm{D}$ zones surface and $D_{\max }$ is the maximum gray level in the image. Using the phenomenological model to be described in this work, the NIOD profile can be transformed into nanometer layers' thickness by an independent calibration method or knowledge of the material scattering properties as shown below.

\subsection{Mathematical model}

The model assumes that the normal or $z$-axis backscattered light intensity from the particles in the deposited layer on the waveguide $x-y$ plane captured by the microscope camera $I_{z}(x, y)=I_{z}$ is given by Eq. (1):

$$
I_{z}(x, y, z)_{\mathrm{det}}=\eta(x, y, z) I_{0}(x, y, z) .
$$

$I_{z}(x, y, z)_{\text {det }}$ is the signal at the camera detector, $\eta(x, y, z)$ is a $z$-direction beam fractional backscattering power with respect to $I_{0}$ from the point $(x, y, z)$ normally towards the microscope objective. Denoting here by $R$ the upper surface reflection constant, we assume phenomenologically, similar to the DELI model, that the elemental $z$-directed differential scattered light intensity $\mathrm{d} I_{z}(x, y, z)$ from the nanoparticles layer towards the detector of the microscope is proportional to $\gamma \times\left(I_{0}(x, y, z)-I_{z}(x, y, z)\right)$ leading to the following differential equation:

$$
\frac{\partial I_{z}}{\partial z}=\gamma\left((1-R) I_{0} \mathrm{e}^{-\alpha z}-I_{z}(x, y, z)\right) .
$$

$\gamma$ is a parameter describing the normally ( $z$-axis only) backscattered light intensity yield of the photons penetrating from the top surface into the deposited material on the substrate. $\alpha$ is the total optical absorption constant of the deposited layer material. Integrating Eq. (2) across the deposited layer from $z=0$ to its film 
thickness contour profile $z=h(x, y)$ at any point $(x, y)$ on the substrate, with the $z$-interface light intensity boundary value $I_{z}(x, y, 0)=0$ gives the following solution:

$$
I_{z}(x, y)=(1-R) I_{0}(x, y) \frac{\gamma}{\gamma-\alpha}\left(\mathrm{e}^{-\alpha h(x, y)}-\mathrm{e}^{-\gamma h(x, y)}\right) .
$$

$I_{0}(x, y)$ is the illuminator beam intensity on the top surface of the sample, see Figs. 1 and 2. In particular, for a homogeneous surface illumination $I_{0}(x, y)=I_{0}$ when the visible optical absorption spectrum of the material is negligible, a condition often met in organic thin layers, Eq. (3) gives a simpler solution similar to that obtained in $[5,10]$ for the DELI evanescent illumination case

$$
I_{z}(x, y)=\left(1-\mathrm{e}^{-\gamma h(x, y)}\right)(1-R) I_{0} .
$$

Moreover, for small $\gamma h<1$ and neglecting reflection, $R \approx 0$, which are experimentally appropriate conditions for our deposited polymer layers in this work, one can further approximate Eq. (4) by a simpler linear Eq. (5):

$I_{z}(x, y)=\left(\gamma h-\frac{1}{2}(\gamma h)^{2}+\frac{1}{6}(\gamma h)^{3} \ldots\right) I_{0}(x, y) \sim \gamma h I_{0}$

The constant $\gamma$ can be determined experimentally by calibrating areas with known thicknesses of the deposited material using AFM or other nanothickness measuring methods using the following relation obtained from Eq. (4):

$$
\gamma=\frac{1}{h\left(x_{r}, y_{r}\right)} \ln \left(1+\frac{I_{z}\left(x_{r}, y_{r}\right)}{I_{0}}\right),
$$

$h\left(x_{r}, y_{r}\right)$ are, independently measured specific calibration points thicknesses at locations $\left(x_{r}, y_{r}\right)$ in the sample and $I_{z}\left(x_{r}, y_{r}\right)$ is the backscattered light intensity from these surface points, normally towards the microscope objective

$$
\begin{aligned}
& h(x, y)=\frac{1}{\gamma} \ln \left(\left(1-\frac{I_{z}(x, y)}{I_{0}}\right)^{-1}\right)= \\
& -\frac{1}{\gamma} \ln \left(1-\left(\frac{I_{z}(x, y)}{I_{0}}\right)\right) .
\end{aligned}
$$

$\gamma$ can be further modelled also as an optical property involving the mean single particle optical scattering cross-section $\sigma_{s}$ and the volume number density of the particulate scatterers $N_{s}$ in the deposited layer. This was done previously for the DELI evanescent technique in [10], which uses the same scattering model and allowing us probing the particles nanosize structural size using Eq. (8):

$$
\gamma=\sigma_{s} \times N_{s} .
$$

The optical image density measurements from the microscope gives us the normalized integrated optical density and since $\eta(x, y, z)$ from Eq. (1) is proportional to NIOD we can write

$$
\frac{\mathrm{NIOD}_{2}\left(h_{2}\right)}{\mathrm{NIOD}_{1}\left(h_{1}\right)} \approx \frac{\eta_{2}}{\eta_{1}}=\frac{1-\mathrm{e}^{-\gamma h_{2}}}{1-\mathrm{e}^{-\gamma h_{1}}} .
$$

Measuring the NIOD's ratio for any two $h_{i}$ in Eq. (9) one can extract the $\gamma$ factor of the material deposited material and from it constructing the thickness height profile of all the observed surface. In fact for thin layers $\gamma h<1$ one can observe that a first approximation exists

$$
\frac{\mathrm{NIOD}_{2}\left(h_{2}\right)}{\mathrm{NIOD}_{1}\left(h_{1}\right)} \approx \frac{h_{2}}{h_{1}},
$$

thus knowledge of one thickness e.g., $h_{1}$ by calibration, helps calculate any other height $h_{2}$ on the surface.

\section{Details of samples preparation}

Chl-a and anthocyanin chromophores extracted by ethanol from red rubin ocimum basilicum (RROB) plant leaves [11] were used in photodeposition experiments of nanolayers onto PMMA, i.e., polymer $\left(\mathrm{C}_{5} \mathrm{H}_{8} \mathrm{O}_{2}\right)_{n}$ substrates. Red leaves of RROB are shown in Fig. 3a. The leaves were macerated and chromophores from the slurry samples were solvated in 2 different solvents using batches of leaves, $10 \mathrm{~g}$ each, in (A) $30 \mathrm{ml}$ distilled $\mathrm{H}_{2} \mathrm{O}$, and (B) $30 \mathrm{ml}$ of $>96 \%$ ethanol in water. Figure $3 \mathrm{~b}$ shows the green color Chl-a chromophore obtained in solution B and Fig. $3 \mathrm{c}$ - the red color anthocyanin chromophore obtained in solution A.

The solutions were used for photodeposition procedures on PMMA substrates using laser irradiation of blue-violet line, $\lambda=405 \mathrm{~nm}$. Only the Chl-a solution irradiation resulted in a photodeposited layer while the anthocyanin solution B created thin layers only by drying the solution drops on the PMMA substrates. Using FTIR we realized that the deposited layer consisted only of PMMA polymer. 5 deposited dots $1 \mathrm{D}$ and 2D profiles obtained by the blue--violet laser photodeposition from solution type $B$ through a proximity mask with circular apertures, each of $3 \mathrm{~mm}$ in diameter, for deposition times in the range of $20-320 \mathrm{~s}$ are shown
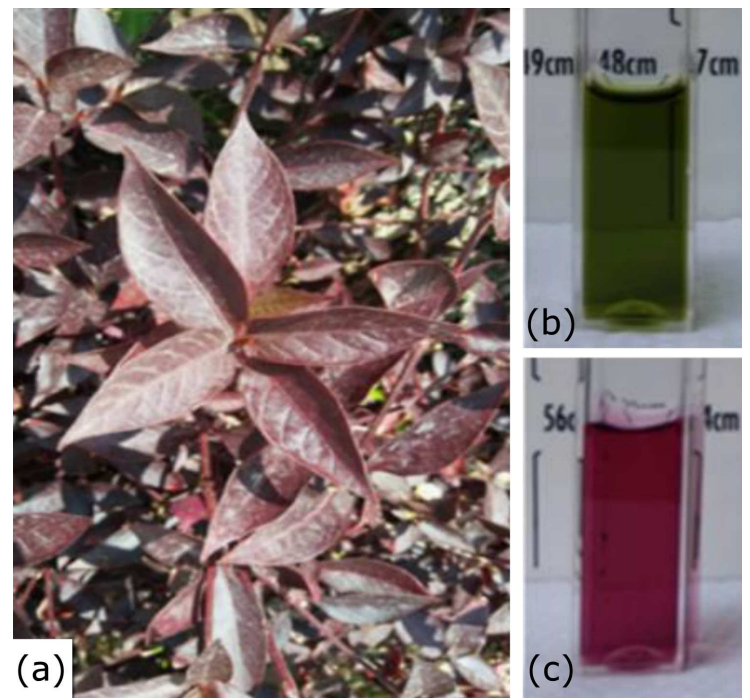

Fig. 3. (a) RROB leaves, (b) cuvette with solution B containing Chl-a, (c) cuvette with solution A containing anthocyanin molecules. 

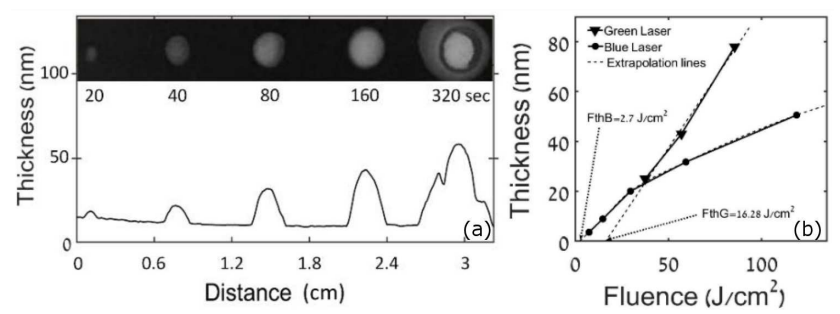

Fig. 4. (a) Top 2D image of 5 deposited dots obtained through a proximity mask with circular apertures, each of $3 \mathrm{~mm}$ in diameter, obtained by increasing the deposition times in the range of $20-320 \mathrm{~s}$ with a blue-violet laser of $\lambda=405 \mathrm{~nm}$ wavelength; below the top image is the dots averaged $1 \mathrm{D}$ thickness profile cross-section plot vs. distance on the same substrate. (b) The 5 dots thickness vs. fluence plot for the blue-violet laser for obtaining the extrapolated fluence threshold values $F_{t h}$ for the blue and green lasers.

in Fig. 4. These time periods correspond to a fluence range of $0-150 \mathrm{~J} / \mathrm{cm}^{2}$. The thickness profiles of the deposition patterns were recorded by capturing images of the optical density obtained from the microscope cameras and the absolute nanometric thicknesses were calculated by the DRLS technique, calibrated from independently measured absolute values, by scanning electron microscopy (SEM). The deposited dots average diameters and also their average film thicknesses increase for longer times of photodeposition. The threshold fluence obtained for the blue-violet laser by extrapolation is about $2.7 \mathrm{~J} / \mathrm{cm}^{2}$ as shown in Fig. $4 \mathrm{~b}$.

\section{3D morphology investigation by DRLS and SEM}

The DRLS technique enables one also to present synthetic 3D profiles images constructed from the $2 \mathrm{D}$ microscope optical images using image processing programs $[12,13]$. For instance, Fig. 5b and Fig. 6 show $3 \mathrm{D}$ nanoscopic details of a smaller sampled area from the 2D image in Fig. 5a. At the periphery of the dot at higher optical magnification a comb type surface deposited pattern with linewidths of about $\approx 20 \mu \mathrm{m}$ (Fig. 5b) displays the blue--violet laser diffraction fringes originating from the narrow illuminated aperture during the photodeposition. These line patterns appear better resolved at the dot edges zones where the photodeposited material is thinnest. The diffraction fringes deposited patterns obtained in Fig. 5b show that the best line resolution observed was about $20 \mu \mathrm{m}$. However, the lines resolution bottleneck was probably limited only by the relatively simple optical system resolution capability used. The theoretical line feature resolution $d$ based on the Rayleigh criterion [14] gives for the optical system experimental photodeposition parameters such as: distance to the mask aperture of $L=24 \mathrm{~mm}$, illuminated mask

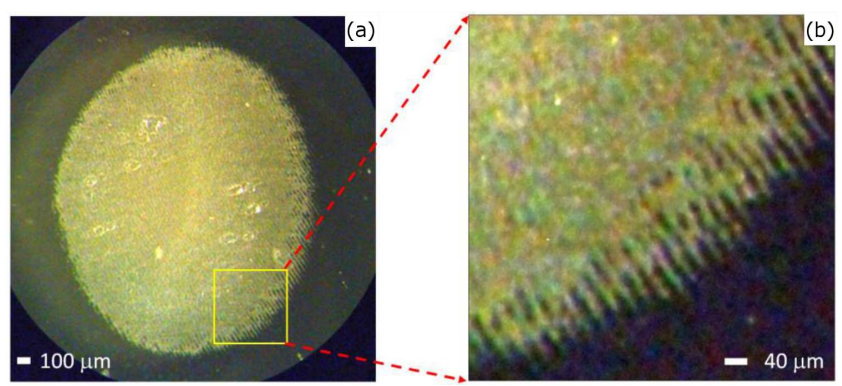

Fig. 5. (a) Optical microscopy image of a photodeposited PMMA dot. (b) Expanded magnified image of the yellow square zone in (a) showing peripheral fringes caused by light diffraction.

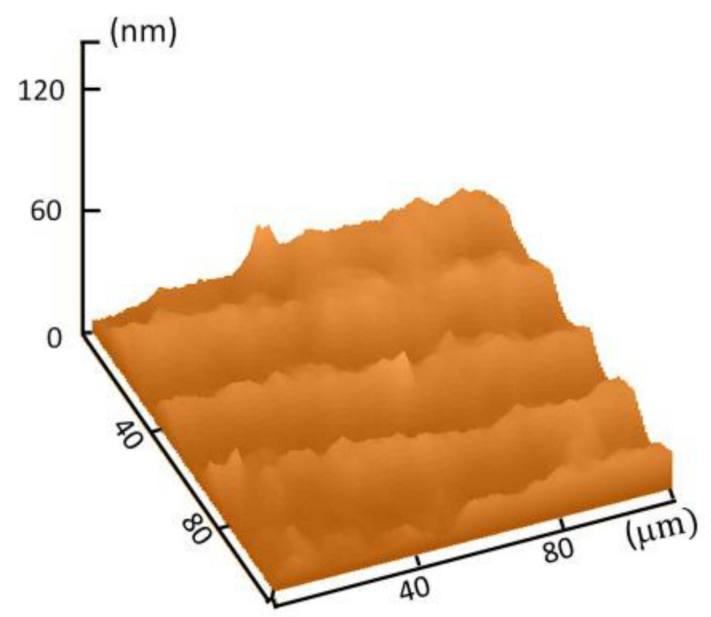

Fig. 6. DRLS synthetic image processed from a sample of the image shown in Fig. 5b.

aperture radius $b=1.5 \mathrm{~mm}$, and laser beam wavelength $\lambda=405 \times 10^{-6} \mathrm{~mm}$, a value quite close to the experimental result shown in Fig. 5b, i.e.:

$$
\begin{aligned}
d=2 \times L \times 1.22 \times \frac{\lambda}{b}=2 \times 24 \times 1.22 \times 405 \times \frac{10^{-6}}{1.5}= \\
0.0158 \mathrm{~mm} \cong 16 \mu \mathrm{m} .
\end{aligned}
$$

For comparison purposes of the DRLS results, Fig. 7 shows typical SEM micrographs at 2 magnifications of the morphology of a similar photodeposited dot layer structure. The images reveal here elongated fibers, lightly twisted with a parallel orientation. The fibers have a mean line width of about $10 \mu \mathrm{m}$ corresponding closely to the results from the DRLS optical investigation shown in Fig. 5 and Fig. 6.

Optical microscopy was performed also for a typical dried drop of anthocyanin red solution A (see Fig. 2c) on a clean PMMA substrate. The as obtained optical DRLS layer morphology shows here also a similarity with those obtained from SEM images (see Fig. 8). 


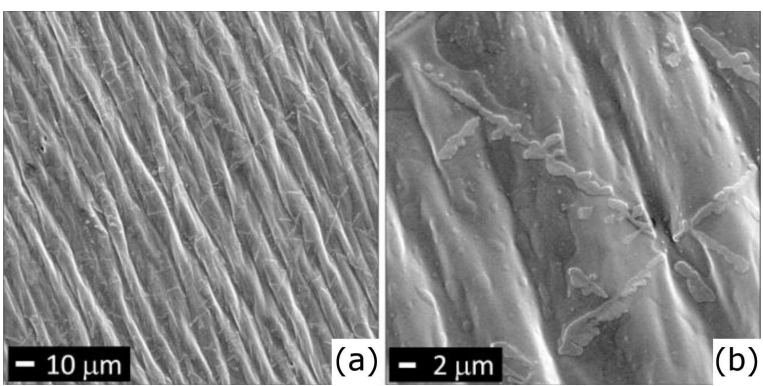

Fig. 7. (a) SEM image of photodeposited PMMA fibers, (b) enlarged image showing anthocyanin strands on the PMMA fibers.
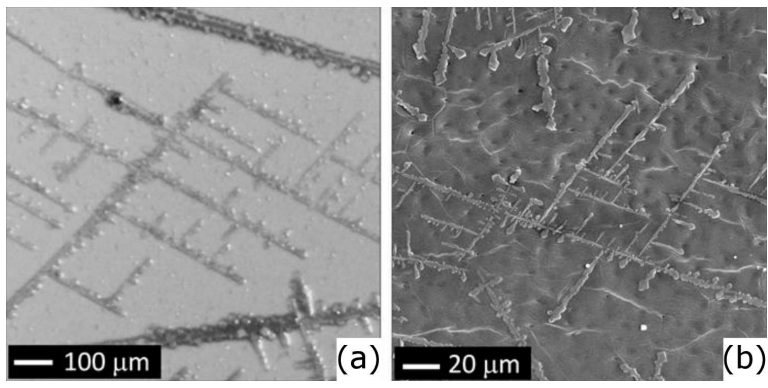

Fig. 8. (a) Optical microscopy of a dried drop from solution B and (b) SEM image of comparable morphology obtained from the layer in Fig. 4 photodeposited during $320 \mathrm{~s}$. The branched needle forms on the top are anthocyanin residues meandering forms layers onto the PMMA substrates.

The experimental value $\gamma=0.974 \times 10^{5} \mathrm{~cm}^{-1}$ obtained in this investigation for PMMA deposition by DRLS is very close to the value $\gamma=0.779 \times 10^{5} \mathrm{~cm}^{-1}$ obtained by the DELI method for nanopolyethylene (PE) layers [10], hinting that in both cases the optical extraction mechanism is the same $z$-directed light scattering phenomenon behavior in organic-dielectric class materials.

\section{Discussion and conclusions}

The DRLS diagnostic technique, based on capturing images of the $z$-axis optical field scattered by the particles nanolayers at maximum contrast is sensitive enough for evaluating nanometric layers up to about $200 \mathrm{~nm}$. It has an advantage over optical transmission microscopy that the optical field observed perpendicular to the substrate surface, has an excellent dark background contrast at a particular oblique illumination angle enabling very sensitive $z$-direction optical microscopy nanometer thickness depth evaluation. This technique is an extended complementary method of the evanescent technique DELI in optical waveguides, developed previously [5], applicable now also to nontransparent substrates. The technique is much easier, economical and non-destructive to use for nanometer films profiling as compared to sophisticated though very expensive scanning methods such as SEM, AFM, SNOM. DRLS is also advantageous for morphology diagnostics of large areas where needed such as in industrial applications.

\section{Acknowledgments}

The authors wish to thank Dr. Igor Lapsker, Dr. Alex Axelevitch and Dr. Boris Apter from HIT for helping with the SEM, FTIR, and laser power calibration measurements, respectively.

\section{References}

[1] A. Poruba, A. Fejfar, Z. Remeš, J. Špringer, M. Vaněček, J. Kočka, J. Meier, P. Torres, A. Shah, J. Appl. Phys. 88, 148 (2000).

[2] R. Apetz, M.P.B. van Bruggen, J. Am. Ceram. Soc. 86, 480 (2003).

[3] M. Hebert, R.D. Hersch, J.-M. Becker, J. Opt. Soc. Am. A 24, 2628 (2007).

[4] J.F. Blinn, Comput. Graph. 16, 21 (1982).

[5] N. Mirchin, B. Apter, I. Lapsker et al., J. Nanosci. Nanotechnol. 12, 2668 (2012).

[6] N. Mirchin, A. Peled, J. Adv. Microsc. Res. 7, 1 (2012).

[7] G. Socol, E. Axente, M. Oane et al., Appl. Surf. Sci. 253, 6535 (2007).

[8] N. Mirchin, I. Lapsker, E. Tannous, A. Peled, Mater. Sci. Appl. 4, 572 (2013).

[9] A.I. Lvovsky, Fresnel Equations, Encyclopedia of Optical Engineering, Taylor and Francis, New York 2013.

[10] N. Mirchin, A. Peled, J. Adv. Microsc. Res. 7, 1 (2012).

[11] U. Szymanowska, U. Złotek, M. Karas, B. Baraniak, Food Chem. 172, 71 (2015).

[12] Irfan Skiljan, IrfanView Graphic Viewer.

[13] Image J, National Institutes of Health (NIH).

[14] C.D. Meinhart, S.T. Wereley, Meas. Sci. Technol. 14, 1047 (2003). 